\title{
Current treatment of peripheral infected wounds: Our vacuum-assisted closure experiences
}

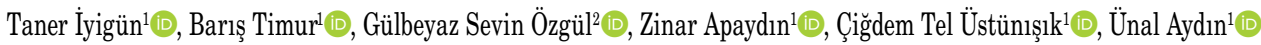 \\ ${ }^{1}$ Department of Cardiovascular Surgery, University of Health Sciences, Istanbul Mehmet Akif Ersoy Thoracic and Cardiovascular Surgery Training and Research Hospital, Istanbul, Turkey \\ ${ }^{2}$ Department of Plastic Reconstructive and Aesthetic Surgery, University of Health Sciences, Istanbul Mehmet Akif Ersoy Thoracic and Cardiovascular Surgery Training and Research \\ Hospital, Istanbul, Turkey
}

\begin{abstract}
Objectives: In this study, we present our experiences on peripheral vacuum-assisted closure (VAC) applications in our clinic.

Patients and methods: Between January 2012 and December 2019, a total of 64 patients (40 males, 24 females; mean age 67.9 years; range, 35 to 94 years) undergoing peripheral VAC therapy were retrospectively analyzed. The VAC system was changed at weekly intervals, until a negative culture was obtained or laboratory values returned to normal ranges and were not suggestive of infections.

Results: In 16 patients (25\%), the main indication for VAC was previous femoral pseudoaneurysm repair. Escherichia coli was the most commonly isolated strain from the wound site. The mean length of hospital stay was 54.1 days.

Conclusion: Peripheral wound infections cause a significant increase in the length of hospital stay, cost, and mortality. Vacuum-assisted closure applications may reduce these rates. It is an ideal method to be used throughout surgery to fight against surgical site infections.
\end{abstract}

Keywords: Cost analysis, negative-pressure wound therapy, peripheral vascular diseases, surgical wound infection.

Vacuum-assisted closure $\left(\mathrm{VAC}^{\circledR}\right)$ is an effective way to remove exudative fluid and to promote wound healing. ${ }^{[1]}$ Currently, VAC therapy has been used in a wide variety of different wound types such as diabetic foot, pressure ulcers, mediastinitis, and amputation-stump sites. ${ }^{[2]}$ The manufacturer of the VAC systems (KCI Medical Inc., TX, USA) do not recommend VAC therapy in patients undergoing vascular reconstruction, for anastomosis sites, or in the presence of exposed vessels. ${ }^{[3]}$ Therefore, the use of VAC therapy in patients with peripheral arterial disease is limited.

Surgical site infections (SSIs) are serious risks for prolonged hospitalizations, morbidity, and mortality. ${ }^{[4]}$ These infections also increase the healthcare costs. ${ }^{[5]}$ The risk of SSI increases particularly in the presence of diseases such as peripheral arterial disease, obesity, and diabetes mellitus $(\mathrm{DM}){ }^{[6]}$ The groin area is particularly risky and surgical incision to this area poses a great risk for SSIs, since this area is a suitable site for bacteria due to its proximity to urogenital and perianal sites. However, it is possible to achieve successful results with VAC therapy with reduced hospital costs.

In this study, we present our experiences on peripheral VAC applications in the treatment of wound infections in our clinic.

\section{PATIENTS AND METHODS}

Between January 2012 and December 2019, a total of 64 patients (40 males, 24 females; mean age 67.9 years; range, 35 to 94 years) undergoing peripheral VAC therapy for wound infections at Istanbul Mehmet Akif Ersoy Thoracic and Cardiovascular Surgery Training and Research Hospital were

Received: December 11, 2019 Accepted: January 23, 2020 Published online: March 10, 2020

Correspondence: Taner İyigün, MD. SBÜ Mehmet Akif Ersoy Göğüs Kalp ve Damar Cerrahisi Eğitim ve Araştırma Hastanesi, Kalp ve Damar Cerrahisi Anabilim Dalı, 34303 Küçükçekmece, İstanbul, Türkiye. e-mail: taneriyi@gmail.com 
retrospectively analyzed. The patient data were obtained from the hospital database. The VAC applications for permanent pacemaker sites and mediastinitis were excluded from the study. A written informed consent was obtained from each patient. The study protocol was approved by the Ethics Committee of Istanbul Mehmet Akif Ersoy Thoracic and Cardiovascular Surgery Training and Research Hospital. The study was conducted in accordance with the principles of the Declaration of Helsinki.

\section{Vacuum-assisted closure therapy protocol}

Patients with superficial or deep tissue infections were primarily treated with wound dressings as recommended by the Department of Aesthetic, Plastic and Reconstructive Surgery of Istanbul Mehmet Akif Ersoy Thoracic and Cardiovascular Surgery Training and Research Hospital. After sufficient treatment, superficial infections were primarily sutured following debridement. Deep and large infections were treated using the VAC. The patients were routinely administered with $3 \mathrm{~g}$ of cephazolin on a daily basis starting with the induction of anesthesia for prophylaxis. Wound cultures were obtained and were debrided under regional anesthesia, if required. If the wound cultures stayed positive or the wound became clinically deteriorated (with increased infectious parameters) despite the treatment, we decided to treat the patient using VAC. The patients were administered with proper antibiotics according to the antibiograms. In case of a negative culture, prophylactic antibiotics were administered. As per the manufacturer's recommendations, we did not use the VAC system for exposed vessels or graft anastomosis sites. The wound was continuously vacuumed at $75 \mathrm{mmHg}$ throughout the therapy. We only used one layer of silver-coated VAC sponges for the wounds. The VAC system was changed at weekly intervals, until a negative culture was obtained or laboratory values including white blood cell count, C-reactive protein, and procalcitonin returned to normal ranges and were not suggestive of any infection. During the VAC changes, we obtained new cultures from the wound site. The wound was also carefully debrided every single time. The Same VAC system was used for every patient (V.A.C. ULTA, KCI USA, Inc., San Antonio, TX, USA). All patients were treated in the hospital throughout the VAC therapy. The wound was primarily closed after removal of the VAC system. In selected cases, the wound was closed using a skin graft.

\section{RESULTS}

In 16 patients (25\%), the main indication for VAC was previous femoral pseudoaneurysm repair (Figure 1). Fourteen patients (21.9\%) had saphenous vein graft site infections. Seventeen patients (26.6\%) developed an infection after different types of femoral interventions (i.e., transcatheter aortic valve implantation,
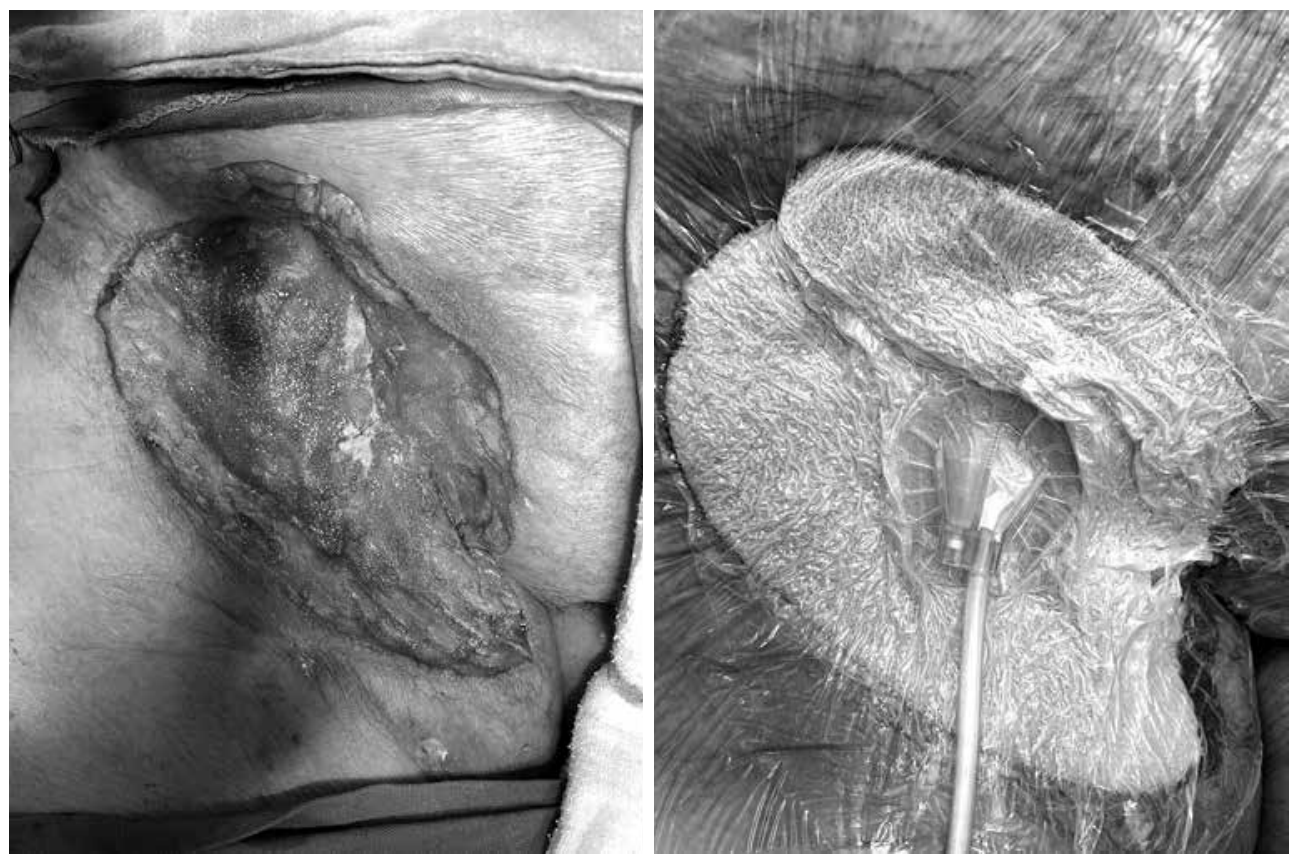

Figure 1. A femoral pseudoaneurysm surgical site infection treated with vacuum-assisted closure therapy. 

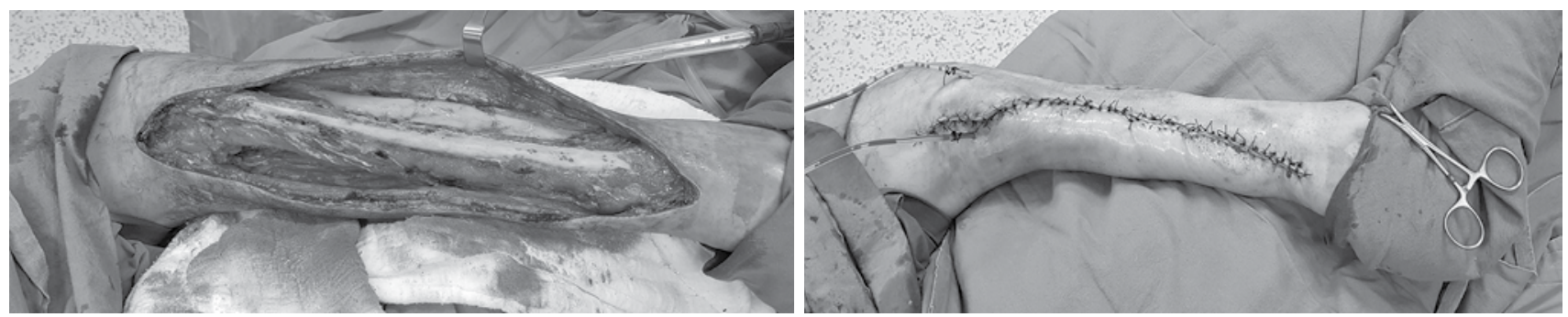

Figure 2. A wound successfully closed up after vacuum-assisted closure therapy.
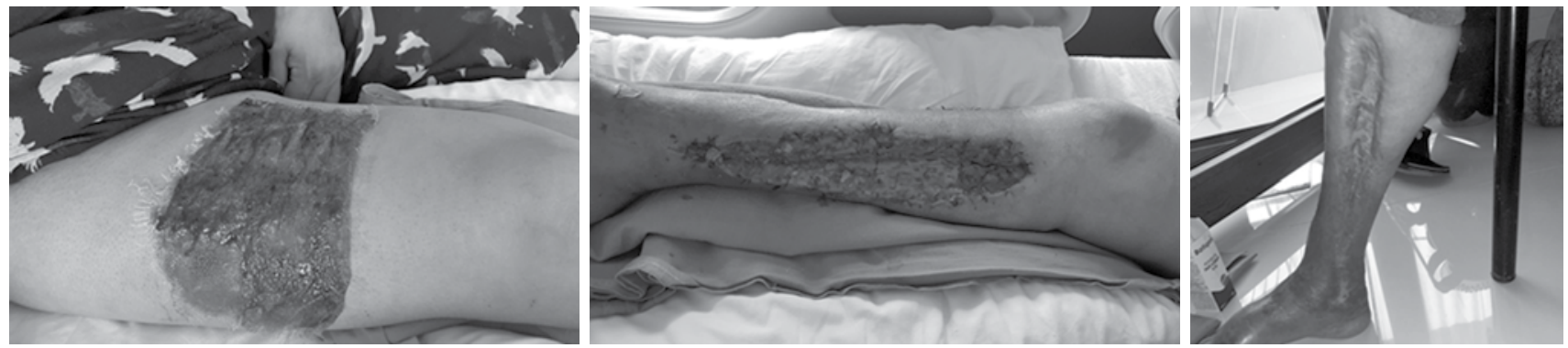

Figure 3. A patient treated successfully using split-thickness skin graft.

arteriovenous fistula repair, and peripheral cannulation for cardiac surgery). Eleven of them (17.2\%) had primary peripheral arterial disease. Two patients (3.1\%) required

\begin{tabular}{lccc}
\multicolumn{3}{l}{ Table 1. Demographic and clinical characteristics of patients } \\
\hline & $\mathrm{n}$ & $\%$ & Mean \\
\hline Age (year) & & & 67.9 \\
Gender & 40 & 62.50 & \\
$\quad$ Male & 24 & 37.50 & \\
$\quad$ Female & & & \\
Causes & 16 & 25 & \\
$\quad$ Femoral pseudoaneursym & 14 & 21.90 & \\
$\quad$ Saphenous vein incision & & & \\
Femoral intervention & 6 & 9.40 & \\
$\quad$ Femoral cannulation & 4 & 6.30 & \\
$\quad$ Femoral infection after ABF & 3 & 4.70 & \\
$\quad$ EVAR & 2 & 3.10 & \\
$\quad$ TAVI & 2 & 3.10 & \\
$\quad$ Femoral AV fistula & & & \\
Comorbidities & 47 & 73.40 & \\
$\quad$ DM & 11 & 17.20 & \\
$\quad$ PAD & & & \\
Microorganism & 18 & 28.1 & \\
$\quad$ Combined & 14 & 21.9 & \\
Escherichia coli & 8 & 12.5 & \\
$\quad$ Enterococcus & & \\
Staphylococcus & & 10.9 & \\
Mean length of hospital stay (days) & & & \\
Mean no of VAC changes & & & \\
Succesfully discharged & 59 & 92.20 & \\
Mortality & 3 & 4.70 & \\
Skin graft & 2 & 3.10 & \\
\hline
\end{tabular}

ABF: Aortobifemoral bypass; EVAR: Endovascular aneursym repair; TAVI: Transcatheter aortic valve implantation; AV: Arteriovenous; DM: Diabetes mellitus; PAD: Peripheral arterial disease; VAC: Vacuum-assisted closure. skin grafts for the wound closure (Figure 2). These patients had deeper wound infections and subcutaneous tissue and muscle debridement were made during the VAC changes. Of the patients, 47 (73.4\%) had DM, which is the most common comorbidity. Demographic and clinical characteristics of the patients are summarized in Table 1.

Escherichia coli was the most commonly isolated strain from the wound site in 14 patients (21.9\%). In 18 patients $(28.1 \%), \geq 2$ bacterial strains were isolated in the cultures. Enterococci, Klebsiella, Pseudomonas, Acinetobacterium, and Candida spp. were the common strains isolated.

The mean length of hospital stay was 54.1 (range, 7 to 150) days. The mean number of VAC changes per patient was 4.9 (range, 1 to 30 ). Fifty-nine of them (92.2\%) were uneventfully discharged from the hospital (Figure 3). Only one patient (1.6\%) in whom VAC therapy was applied to the amputation stump a major bleeding. Therapy was immediately ceased and the patient was scheduled for an emergency surgery. Bleeding was controlled during surgery. Among all patients, three of them (4.7\%) died, and all were due to septic complications. One of the non-survivors (1.6\%) had Acinetobacter spp. infection at the time of admission.

\section{DISCUSSION}

In the present study, we treated our patients with wound infections with VAC therapy and our success 
rate was $92 \%$ which is relatively higher than reported success rates in the literature. ${ }^{[7]}$ Diabetes mellitus was the most common comorbidity in our patient group and the groin area was the most common infection site. In previous studies, the intestinal flora was the most common site. ${ }^{[7]}$

Studies have demonstrated that VAC systems enhance wound healing with multiple effects such as increasing blood flow, reducing edema, and promoting granulation tissue formation. ${ }^{[8]}$ These systems also promote angiogenesis and endothelial proliferation. ${ }^{[8]}$ Therefore, VAC therapy is an effective way to treat deep tissue wounds. Particularly in patients with DM and peripheral arterial diseases, VAC is helpful for extremity salvage. Despite these positive effects, VAC therapy should not be considered as an alternative treatment to surgery. Surgical debridement is still the first choice of treatment. Surgical revision is the key for wound healing. The VAC therapy should be used as an adjunct treatment.

Diabetes is one of the most important risk factors for peripheral wounds and it complicates existing wounds and makes it longer to heal. Diabetes significantly disturbs proper wound healing and tissue regeneration, leading to peripheral arterial disease. ${ }^{[9]}$ Elevated systemic glucose levels in diabetic patients cause both microvascular and macrovascular complications which alters angiogenesis. ${ }^{[10]}$ In addition, DM increases the risk for infection. Approximately one-fourth of all diabetic patients suffer from lower extremity infections in their life, and some of them end with amputations. ${ }^{[11]}$ Diabetes mellitus is an independent risk factor for amputation, as well. ${ }^{[2]}$ Therefore, it is one of the greatest risks in wound healing. In our study, $73.4 \%$ of the patients were diabetic, as expected. Strict glucose control is vital in these patients. Endocrinologists should be a part of the treatment strategy.

The VAC manufacturers do not recommend VAC therapy in patients undergoing vascular reconstruction, for anastomosis sites, or in the presence of exposed vessels. ${ }^{[3]}$ In our study, we only had one patient with major bleeding. Different series have different numbers of bleedings. Svensson et al. ${ }^{[7]}$ reported two major bleeding cases. Brehm et al. ${ }^{[12]}$ also reported heavy bleeding in their series. The most common reason for heavy bleeding is because of the negative suction of the system. Exposed vessels or graft anastomosis sites were the most common sites of bleeding. In our patient, it was one of the exposed vessels after amputation. In high-risk patients, if VAC is needed, non-adhesive silicone-based sponges should be placed before the silver-coated adhesive sponges to prevent such an undesired complication.

A non-healing wound is strongly associated with higher mortality and amputation rates. Therefore, wound healing is critical. ${ }^{[11]}$ Even with VAC therapy, one-year mortality may be as high as $27 \% .^{[2]}$ Traditional methods such as wound dressings and irrigation can be effective ways of treating SSIs. However, these methods are mostly applied in the hospital setting which may cause cross-contamination between the patients. The VAC systems, on the other hand, are changed in the operation room setting in our hospital, which provides a more sterile environment for the wound. Therefore, we choose using VAC systems in a short manner of time.

Staphylococcus aureus (S. aureus), Streptococci, Gramnegative bacteria, and anaerobic bacteria are mostly reproduced in the cultures of patients with peripheral wound infections. ${ }^{[13]}$ A study from Turkey showed that $S$. aureus and Streptococci infections were the most common culprits. Escherichia coli (E. coli) and Pseudomonas spp. were also the most common Gramnegative sources of infection. ${ }^{[14]}$ About $24 \%$ of bacterial infections were multidrug-resistant strains. ${ }^{[14]}$ Crosscontamination during the hospital stay is a major risk for patients. A total of $28 \%$ of the patients were infected by more than two different strains. Some of them were multi-drug resistant strains, leading to longer hospital stays. In our study, E. coli was the most commonly isolated strain. We observed that the most common VAC site was the groin after femoral pseudoaneurysm repair. The possible reason for this is the area's proximity to the urogenital organs and anal area. The close proximity to these sites increases the risk for infection, as these sites are rich in the intestinal and urogenital floral bacteria. The personal hygiene is also of utmost importance for these patients. Escherichia coli as the most commonly isolated strain in our study supports this claim. Aggressive antibiotherapy in these patients are needed. In our routine practice, we used cephazolin 3 daily for surgical prophylaxis, starting with induction of anesthesia. According to the antibiograms, we modify the treatment abruptly.

Split-thickness skin grafting (STSG) helps stable and durable wound closure. ${ }^{[15]} \mathrm{It}$ is associated favorable outcomes in different patient populations such as diabetic foot. ${ }^{[16]}$ In our study, three patients needed skin grafts. All of them were successfully discharged from the hospital without any additional complications. In our study, most of the patients did not need STSG. 
We prefer to use VAC system as early as possible which enables us to primarily close the wound. There are studies showing considerable differences in favor of the VAC followed by STSG. In the patients scheduled to be treated using a skin graft, VAC before the treatment should be employed. ${ }^{[17]}$

Cost effectivity of the VAC therapy is another issue on the matter. Philbeck et al ${ }^{[18]}$ reported that VAC therapy had lower costs due to much faster healing time. In our study, the mean length of hospital stay was 54.1 days, which was longer. However, to have cost-effective treatments, patients with VAC systems attached can be discharged earlier and they may later apply to the clinics for VAC changes weekly to shorten the hospital stay. Pinocy et al. ${ }^{[19]}$ suggested the possibility of wither continuing the VAC therapy outside the hospital or that, after 14 days of treatment, the wound may be closed performing a secondary skin suture in a vertical mattress fashion. In our clinic, we do not have a procedure for follow-up of patients outside the hospital with a VAC system. Therefore, all patients were followed in the inhospital setting. Discharging a patient with VAC system attached may have complications such as the drape of the VAC system opened, particularly in the folding areas of the skin (particularly in the groin area), which may cause additional infections. It should be taken into consideration. However, following them outside the hospital surely further reduce the treatment-related costs.

Pain in these patients is another aspect to be evaluated. Wounds to be treated with VAC are painful in nature. Most of them are infected wounds or ulcers. The VAC system itself may cause pain due to negative pressure it applies. However, there is no reported study on this topic. Strategies to minimize pain should be employed. ${ }^{[20]}$

The main limitation to this study is its retrospective nature. In addition, patients had a wide variety of different SSIs in our study. Therefore, further large-scale, prospective studies would provide more accurate results in terms of wound healing, quality of life, and cost effectivity of the VAC therapy.

In conclusion, peripheral wound infections are associated with significantly longer length of hospital stay, and increased cost and mortality. The VAC applications may reduce these rates. Most hospital infections are multi-drug resistant. The VAC systems increase blood flow, reduces edema, and promotes granulation tissue. It is an ideal method to be used during surgery to fight with SSIs.

\section{Declaration of conflicting interests}

The authors declared no conflicts of interest with respect to the authorship and/or publication of this article.

\section{Funding}

The authors received no financial support for the research and/or authorship of this article.

\section{REFERENCES}

1. Morykwas MJ, Argenta LC, Shelton-Brown EI, McGuirt W. Vacuum-assisted closure: a new method for wound control and treatment: animal studies and basic foundation. Ann Plast Surg 1997;38:553-62.

2. Nordmyr J, Svensson S, Björck M, Acosta S. Vacuum assisted wound closure in patients with lower extremity arterial disease. The experience from two tertiary referralcentres. Int Angiol 2009;28:26-31.

3. V.A.C. Therapy Clinical Guidelines- A reference source for clinicians. 2-B-128-CA-EN Rev B 12/2014. Available at: https://www.acelity.com/-/media/Project/Acelity/ Acelity-Base-Sites/shared/PDF/2-b-128-ca-enb-clinicalguidelines---canada---en.pdf

4. Turtiainen J, Saimanen E, Partio T, Kärkkäinen J, Kiviniemi V, Mäkinen K, et al. Surgical wound infections after vascular surgery: prospective multicenter observational study. Scand J Surg 2010;99:167-72.

5. de Lissovoy G, Fraeman K, Hutchins V, Murphy D, Song D, Vaughn BB. Surgical site infection: incidence and impact on hospital utilization and treatment costs. Am J Infect Control 2009;37:387-97.

6. Murphy P, Lee K, Dubois L, DeRose G, Forbes T, Power A. Negative pressure wound therapy for highrisk wounds in lower extremity revascularization: study protocol for a randomized controlled trial. Trials 2015;16:504.

7. Svensson S, Monsen C, Kölbel T, Acosta S. Predictors for outcome after vacuum assisted closure therapy of perivascular surgical site infections in the groin. Eur $J$ Vasc Endovasc Surg 2008;36:84-9.

8. Schintler MV. Negative pressure therapy: theory and practice. Diabetes Metab Res Rev 2012;28:72-7.

9. Okonkwo UA, DiPietro LA. Diabetes and Wound Angiogenesis. Int J Mol Sci 2017;18. pii: E1419.

10. Altabas V. Diabetes, Endothelial Dysfunction, and Vascular Repair: What Should a Diabetologist Keep His Eye on? Int J Endocrinol 2015;2015:848272.

11. Albrant D. Management of foot ulcers in patients with diabetes. Am Pharm Assoc 2000;40:467-74.

12. Brehm V, Steenvoorde P, Oskam J. Regarding "Preservation of infected and exposed vascular grafts using vacuum assisted closure without muscle flap coverage". J Vasc Surg 2006;44:226.

13. Roberts AD, Simon GL. Diabetic foot infections: the role of microbiology and antibiotic treatment. Semin Vasc Surg 2012;25:75-81.

14. Aysert Yıldız P, Özdil T, Dizbay M, Güzel Tunçcan Ö, Hizel K. Peripheral arterial disease increases the risk of 
multidrug-resistant bacteria and amputation in diabetic foot infections. Turk J Med Sci 2018;48:845-50.

15. Naz I, Walters ET, Janhofer DE, Penzler MM, Tefera EA, Evans KK, et al. Outcomes of Split-thickness Skin Grafting for Foot and Ankle Wounds in Patients With Peripheral Arterial Disease. Wounds 2019;31:272-8.

16. Rose JF, Giovinco N, Mills JL, Najafi B, Pappalardo J, Armstrong DG. Split-thickness skin grafting the high-risk diabetic foot. J Vasc Surg 2014;59:1657-63.

17. Saaiq M, Hameed-Ud-Din, Khan MI, Chaudhery SM. Vacuum-assisted closure therapy as a pretreatment for split thickness skin grafts. J Coll Physicians Surg Pak 2010;20:675-9.
18. Philbeck TE Jr, Whittington KT, Millsap MH, Briones RB, Wight DG, Schroeder WJ. The clinical and cost effectiveness of externally applied negative pressure wound therapy in the treatment of wounds in home healthcare Medicare patients. Ostomy Wound Manage 1999;45:41-50.

19. Pinocy J, Albes JM, Wicke C, Ruck P, Ziemer G. Treatment of periprosthetic soft tissue infection of the groin following vascular surgical procedures by means of a polyvinyl alcohol-vacuum sponge system. Wound Repair Regen 2003;11:104-9.

20. Lambert KV, Hayes P, McCarthy M. Vacuum assisted closure: a review of development and current applications. Eur J Vasc Endovasc Surg 2005;29:219-26. 